\title{
Nanomaterials definition matters
}

\author{
The definition and classification of nanomaterials in regulations leave too much room for interpretation.
}

$\mathrm{D}$ efining what we mean by a nanomaterial is never straightforward. For some, the size of the material should be a few nanometres, for others it should be smaller than a few tens of nanometres, for still others anything less than a micrometre will do. Also, for some, one dimension at the nanoscale is enough; for others it should be at least two or even all three.

For different compounds, the properties that distinguish a nanoscale specimen from its bulk correspondent occur at different sizes. The transition is rarely abrupt, and the properties evolve from bulk to nanoscale in a continuous way, so that establishing a threshold size is arbitrary. Finally, the physical and chemical properties of nanomaterials, as well as environmental and health toxicity, depend on the precise shape and composition as well as size.

All the above elements make finding a universal definition and classification an impossible task, especially if compared with chemicals that can in principle be classified just according to their formula. This presents serious challenges to regulations of products that include nanomaterials, especially in light of the real or perceived risks that nanomaterials pose to the environment or to human health. In a Perspective article in this issue, Antonia Praetorius and colleagues examine the problems with the nanomaterials definition and classification in regulations, focusing in particular on EU documents.

The main documents analysed are the European Commission's so-called Recommendation 2011 (which established non-binding guidelines for the classification of nanomaterials), the Novel Food Regulation, the Cosmetics Regulation and the Biocide Regulation. A focus of all definitions in these documents is the size range between $1 \mathrm{~nm}$ and $100 \mathrm{~nm}$. Because the nanoparticles present in a material are distributed in size, an additional challenge for regulations is deciding how many of the particles should fall within the defined criteria for the materials to be classified as a nanomaterial. Both the Recommendation 2011 and the Biocide Regulation describe a nanomaterial as a material that contains at least $50 \%$ of the particles (by number) in the $1-100 \mathrm{~nm}$ range. Because of the difficulties in reliably quantifying components at the lower end of that range, however, such a definition can easily lead to errors. For this reason, the authors suggest adopting a weight-based threshold for the size distribution, as applied for example by the US Environmental Protection Agency, rather than a number-based approach.

Perhaps most importantly, Praetorius and colleagues emphasize the presence of terms in definitions that can lead to freedom of interpretation and therefore difficulty in law application: for example the use of the terms "insoluble" or "biopersistent" to define nanomaterials in the Cosmetics Regulation, which is too ambiguous. Furthermore, the classification as nanomaterials of particles larger than $100 \mathrm{~nm}$ that retain properties characteristic of the nanoscale means very little without a definition of what those properties are.

Very likely, a degree of ambiguity in the definition and classification of nanomaterials will always persist. For scientific purposes this is not necessarily a major problem, provided that both researchers involved in a study and colleagues in the field understand each other. But for regulatory purposes it is problematic because defining an effective and reliable framework is essential for a successful (and safe) use of nanomaterials outside research. The ultimate solution may rely on abandoning generic definitions of nanomaterials and focus on the specific properties and potential hazard of every substance included in a product, as determined by their chemical composition, shape and size. Achieving this for every substance is unfeasible at present, so analyses and suggestions like those by Praetorius and colleagues are the best way forward.

Published online: 5 March 2019

https://doi.org/10.1038/s41565-019-0412-3

\section{Recognizing peer reviewers}

Nature Nanotechnology has started publicly acknowledging reviewers of published papers.

$\mathrm{B}$ y scrolling to the end of the Nature Nanotechnology papers published online since the middle of February 2019, our readers will notice that we now thank some reviewers by name. Nature took this initiative about three years ago, and a number of other Nature journals, including Nature Nanotechnology, are now following its example.

To be clear, we are still committed to protecting the identity of reviewers if they do not wish to be named. Only once we have taken the decision to publish a paper do we contact reviewers and ask whether they agree to be acknowledged publicly. Should they not agree or simply not respond, we would acknowledge them anonymously. At Nature, about $80 \%$ of papers published since the trial began have at least one reviewer named. More specifically, about 55\% of reviewers opted in, $25 \%$ of them opted out and $20 \%$ did not respond.

The trial is one of several initiatives launched by the Nature journals to improve transparency in the assessment and reporting of scientific advances, like the publication of the reviewer reports at Nature Communications or the use of reporting summaries in specific fields of research.
Perhaps more importantly, it is a simple way to recognize publicly the expert contribution that is vital in the editorial decisions we make. The comments of reviewers can be invaluable in corroborating the validity of a study and helping the authors of a paper to strengthen their results. The purpose of peer review is to improve science, and for doing that, our reviewers deserve our thanks.

Published online: 5 March 2019 https://doi.org/10.1038/s41565-019-0413-2 